\title{
Effect of taurine on the proliferation and apoptosis of human hepatocellular carcinoma HepG2 cells
}

\author{
SHUO TU $^{1 *}$, XIALI ZHANG ${ }^{2 *}$, DAYA LUO $^{1}$, ZHUOQI LIU $^{1}$, XIAOHONG YANG $^{1}$, \\ HUIFANG WAN ${ }^{3}$, LEHAN YU ${ }^{3}$, HUA $^{3}$ and FUSHENG WAN ${ }^{1}$ \\ ${ }^{1}$ Department of Biochemistry and Molecular Biology, Basic Medical College of Nanchang University; \\ ${ }^{2}$ Department of Experimental Animals; ${ }^{3}$ Medical Experiment and Teaching Department, \\ Nanchang University, Nanchang, Jiangxi 330006, P.R. China \\ Received June 16, 2014; Accepted February 4, 2015
}

DOI: $10.3892 / \mathrm{etm} .2015 .2476$

\begin{abstract}
The aim of the present study was to observe the effect and molecular mechanism of taurine (Tau) on the cell proliferation and apoptosis of human hepatocellular carcinoma (HHCC) HepG2 cells. HHCC HepG2 cells were used as target cells, and the cell survival rate was assessed using a multi-time-step method. The p53 upregulated modulator of apoptosis (PUMA) gene was transiently transfected by lipofection and subsequently silenced with specific small interfering (si)RNA. The cell apoptosis rate was detected by flow cytometry, and protein expression levels were analyzed with western blotting. Addition of 20-160 mM Tau was shown to have a significant inhibitory effect on cell proliferation, while promoting the induction of HHCC HepG2 cell apoptosis $(\mathrm{P}<0.05)$. Transfection of the PUMA gene significantly enhanced the ability of Tau to inhibit proliferation and induce apoptosis of HepG2 cells. In addition, transfection of the PUMA gene increased the protein expression of B-cell lymphoma-2-associated $X$ and reduced the expression of $\mathrm{B}$-cell lymphoma-2 $(\mathrm{P}<0.05)$. Silencing the PUMA gene with specific siRNA was demonstrated to significantly reduce the ability of Tau to inhibit proliferation and induce the apoptosis of HHCC HepG2 cells $(\mathrm{P}<0.01)$. Therefore, the PUMA gene was shown to have an important role in mechanism underlying the effect that Tau exerts on cell proliferation and apoptosis in HHCC HepG2 cells.
\end{abstract}

Correspondence to: Dr Fusheng Wan, Department of Biochemistry and Molecular Biology, Basic Medical College of Nanchang University, 461 Bayi Avenue, Nanchang, Jiangxi 330006, P.R. China E-mail: fushengwandoc@163.com

*Contributed equally

Key words: taurine, human hepatocellular carcinoma HepG2 cells, p53 upregulated modulator of apoptosis, apoptosis, gene expression

\section{Introduction}

Carcinomas are one of the most detrimental human diseases, and malignant tumors reduce the quality of life and are the leading cause of mortality in China (1). Liver cancer is one of the most common types of cancer, with the highest mortality rate among malignant tumors $(2,3)$. Liver cancer is considered a digestive system tumor with a high malignant potential and poor prognosis, and the majority of patients succumb within a few weeks or months following diagnosis (4). The prevention, control, diagnosis and treatment of liver cancer have become important subjects within healthcare research, and replacing or improving conventional treatment methods for liver cancer is vital. Gene therapy, as an efficient, specific and highly targeted treatment, has received increasing attention $(5,6)$.

Taurine (Tau) is a sulfur-containing acid with an amino group, that has the ability to scavenge oxygen-free radicals, regulate intracellular calcium homeostasis, maintain cell membrane stability and protect cells (7). The molecule has been found to be particularly effective in the prevention of cardiovascular and cerebrovascular diseases (8). A previous study also demonstrated antitumor properties of Tau (9). Furthermore, the addition of a certain amount of Tau to drinking water has been shown to extend the mean lifespan of mice with transplanted tumors, with a tumor growth inhibition rate of $42.26 \%$ (10). Additionally, serum Tau levels in patients with breast cancer have been found to be significantly lower compared with those in high-risk and control groups. Thus, Tau may become a novel indicator for the early diagnosis of breast and bladder cancers (11-13). The study of tumor prevention and treatment with Tau is nascent. However, research into the effect of Tau on tumors remains limited, and the mechanism underlying the antitumor ability of Tau is yet to be elucidated. Biochemical analysis has revealed that expression of the proapoptotic protein, B-cell lymphoma-2-associated X (Bax), is enhanced following treatment with various doses of Tau, whereas expression of the antiapoptotic protein, B-cell lymphoma-2 (Bcl-2), is inhibited in S180 transplanted tumor nude mice. Furthermore, apoptotic cells can be observed morphologically following Tau treatment (14). Tau can also function as an antitumor agent by downregulating the expression of matrix metalloproteinase-2, upregulating 
$\mathrm{N}$-acetylgalactosaminyltransferase, and inhibiting the potential invasion and metastasis induced by ionizing radiation (13). In a previous study, Tau was shown to induce the apoptosis of human colon cancer cells by upregulating the expression of the p53 upregulated modulator of apoptosis (PUMA) gene, independent of p53 (15). However, the ability of Tau to prevent liver cancer has yet to be documented.

In the present study, human hepatocellular carcinoma (HHCC) HepG2 cells were used as target cells, in which the effect and molecular mechanism of Tau on cell proliferation and apoptosis was observed. The aim of the present study was to provide novel targets and targeting drugs for the prevention of liver cancer.

\section{Materials and methods}

Cells and drug handling. HHCC HepG2 cells were obtained from the Cell Bank of the Type Culture Collection of the Chinese Academy of Sciences Committee (Shanghai, China). The cells were cultured in vitro in RPMI 1640 culture medium containing $10 \%$ fetal bovine serum (FBS; Gibco Life Technologies, New York, NY, USA) at $37^{\circ} \mathrm{C}$ and $5 \% \mathrm{CO}_{2}$. Cells in the logarithmic phase were divided randomly into a control group, Tau treatment groups (with concentrations of 20, 40, 80 and $160 \mathrm{mM} \mathrm{Tau}$ ) and a cisplatin (DDP) treatment group (10 $\mu \mathrm{g} / \mathrm{ml}$ DDP). The effect of Tau (Sigma-Aldrich, St. Louis, MO, USA) on HepG2 cell proliferation was observed after 24, 48 and $72 \mathrm{~h}$.

Survival rate detection. Logarithmic-phase cells were collected and dyed with Trypan blue (Shanghai Biological Technology Co., Ltd., Shanghai, China) to monitor and adjust the concentration of the cell suspension. When cells are dyed with Trypan blue, living cells appear transparent, whereas dead cells are dyed blue. A $200-\mu 1$ cell suspension was added to each well of a 96-well plate, and the cell density was adjusted to 3,000-8,000 cells/well. Each group was assessed in quadruplicate. The plates were incubated at $37^{\circ} \mathrm{C}$ and $5 \% \mathrm{CO}_{2}$ for $24 \mathrm{~h}$. Following addition of the drugs, the plates were incubated for 24, 48 and $72 \mathrm{~h}$. At the end of each incubation period, $20 \mu \mathrm{l}$ CellTiter 96 ${ }^{\circledR}$ AQueous One Solution Reagent (Promega Corporation, Madison, WI, USA) was added to each well, and the plates were incubated for an additional $4 \mathrm{~h}$. A microplate reader (xMark ${ }^{\mathrm{TM}}$; Bio-Rad Laboratories, Inc., Hercules, CA, USA) was used to detect and record the optical density (OD) of each well using a wavelength of $490 \mathrm{~nm}$, and the inhibition rate was calculated as follows: Inhibition rate $(\%)=(1-$ OD of the experimental group/OD of the control group) x 100 .

Apoptosis evaluation. Cells were seeded in a 50-ml culture flask for $24 \mathrm{~h}$, and following treatment with the variable concentrations of the drugs for $48 \mathrm{~h}$, non-EDTA pancreatin (Sigma-Aldrich) was used to digest and collect the cells. The cells were washed twice with cold phosphate-buffered saline (PBS) and centrifuged at 1,000 x $\mathrm{g}$ for $5 \mathrm{~min}$, after which $1-5 \times 10^{5}$ cells were collected. The cells were resuspended in $400 \mu \mathrm{l} 1 \mathrm{X}$ annexin-V binding buffer, and $5 \mu \mathrm{l}$ annexin V-fluorescein isothiocyanate (FITC) staining solution (BestBio, Shanghai, China) was added and gently mixed. The cells were incubated in the dark on ice for $15 \mathrm{~min}$. Next,
$10 \mu l$ propidium iodide (PI) staining solution (BestBio) was added and mixed evenly with the cells. Finally, the cells were incubated in the dark on ice for $5 \mathrm{~min}$, and within $1 \mathrm{~h}$, the rate of cell apoptosis was detected by flow cytometry (FCM) using a BD FACSCalibur (Becton Dickinson Biosciences, Franklin Lakes, NJ, USA).

Western blotting. The cells were washed with PBS and lysed with radioimmunoprecipitation assay cell lysis reagent, containing proteinase and phosphatase inhibitors (Solarbio Science \& Technology Co., Ltd., Beijing, China) at $4^{\circ} \mathrm{C}$ for $30 \mathrm{~min}$. The total protein was collected for sodium dodecyl sulfate-polyacrylamide gel electrophoresis. The protein concentrations were determined used the bicinchoninic acid protein assay reagent (Pierce, Rockford, IL, USA) according to the manufacturer's instructions. The total protein content of each well was $30 \mu \mathrm{g}$. A wet transfer method was used to transfer the protein to the polyvinylidene fluoride membrane (EMD Millipore Corporation, Billerica, MA, USA). The membrane was incubated with polyclonal antibodies against PUMA (ab9643; Abcam, Cambridge, UK), Bax (sc-526; Santa Cruz Biotechnology, Inc., Dallas, TX, USA) and Bcl-2 (sc-783; Santa Cruz Biotechnology), and a monoclonal antibody against $\beta$-actin (TA-09; Santa Cruz Biotechnology, Inc.). Following incubation overnight at $4{ }^{\circ} \mathrm{C}$, the membrane was complexed with a horseradish peroxidase-conjugated secondary antibody (ZSGB-BIO, Beijing, China) for $1.5 \mathrm{~h}$. An enhanced chemiluminescence immunological method was performed using a kit (Pierce) for detection in a dark room. Image-Pro ${ }^{\circledR}$ Plus 6.0 gel image analysis software (Media Cybernetics, Inc., Rockville, MD, USA) was used to scan the gray values of the protein bands, and the $\beta$-actin internal reference bands were used as a control to perform the semi-quantitative analysis.

Effect of exogenous PUMA transfection on apoptosis. HepG2 cells were collected in the logarithmic phase and randomly divided into six groups. In the control group, the culture medium was removed and replaced with serum-free medium, and the cells were cultivated for $6 \mathrm{~h}$, after which the complete medium was restored for a 48-h cultivation. In the negative control group (HA group), the cells were transfected with the empty vector, $\mathrm{pCEP} 4-(\mathrm{HA})_{2}-\mathrm{C} 1$. In the positive control group (DDP group), the cells were treated with $10 \mu \mathrm{g} / \mathrm{ml}$ DDP. The experimental group was subdivided into the pCEP4-(HA) $)_{2}$-PUMA group (plasmid was provided by Professor Yu Jian, University of Pittsburgh Cancer Institute, Pittsburgh, PA, USA), the Tau $160 \mathrm{mM}$ group (treated with $160 \mathrm{mM}$ Tau for $48 \mathrm{~h}$ ), the pCEP4-(HA) $)_{2}$-PUMA+Tau 160 mM group (transiently transfected with recombinant vector pCEP4-(HA) $)_{2}$ PUMA for $6 \mathrm{~h}$, after which the solution was changed and $160 \mathrm{mM}$ Tau was added.

The pCEP4-(HA) $)_{2}$-PUMA recombinant plasmid and pCEP4-(HA) $)_{2}-\mathrm{Cl}$ empty vector plasmid were transfected into the HHCC HepG2 cells using a Lipofectamine 2000 (Invitrogen Life Technologies, Carlsbad, CA, USA) in vitro DNA-mediated transfection reagent.

Effect of PUMA-specific small interfering (si)RNA on apoptosis. Based on human PUMA cDNA sequences, the siRNA fragments, 5'-GGGUCCUGUACAAUCUCAUTT-3' 


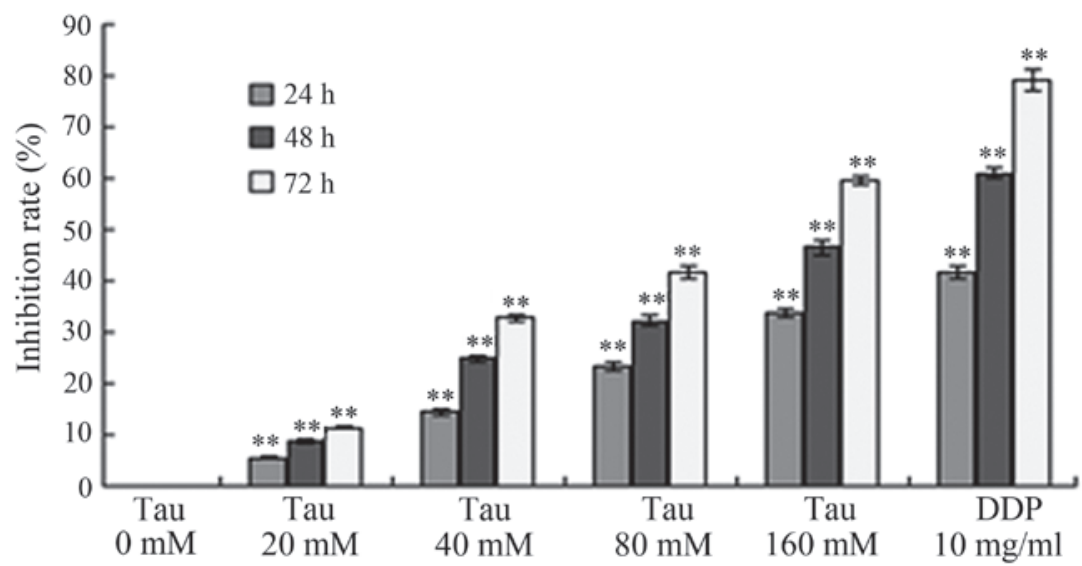

Figure 1. Effect of Tau on the inhibition rate of HepG2 cell proliferation. ${ }^{* *} \mathrm{P}<0.01$, vs. control group. Tau, taurine; DDP, cisplatin.
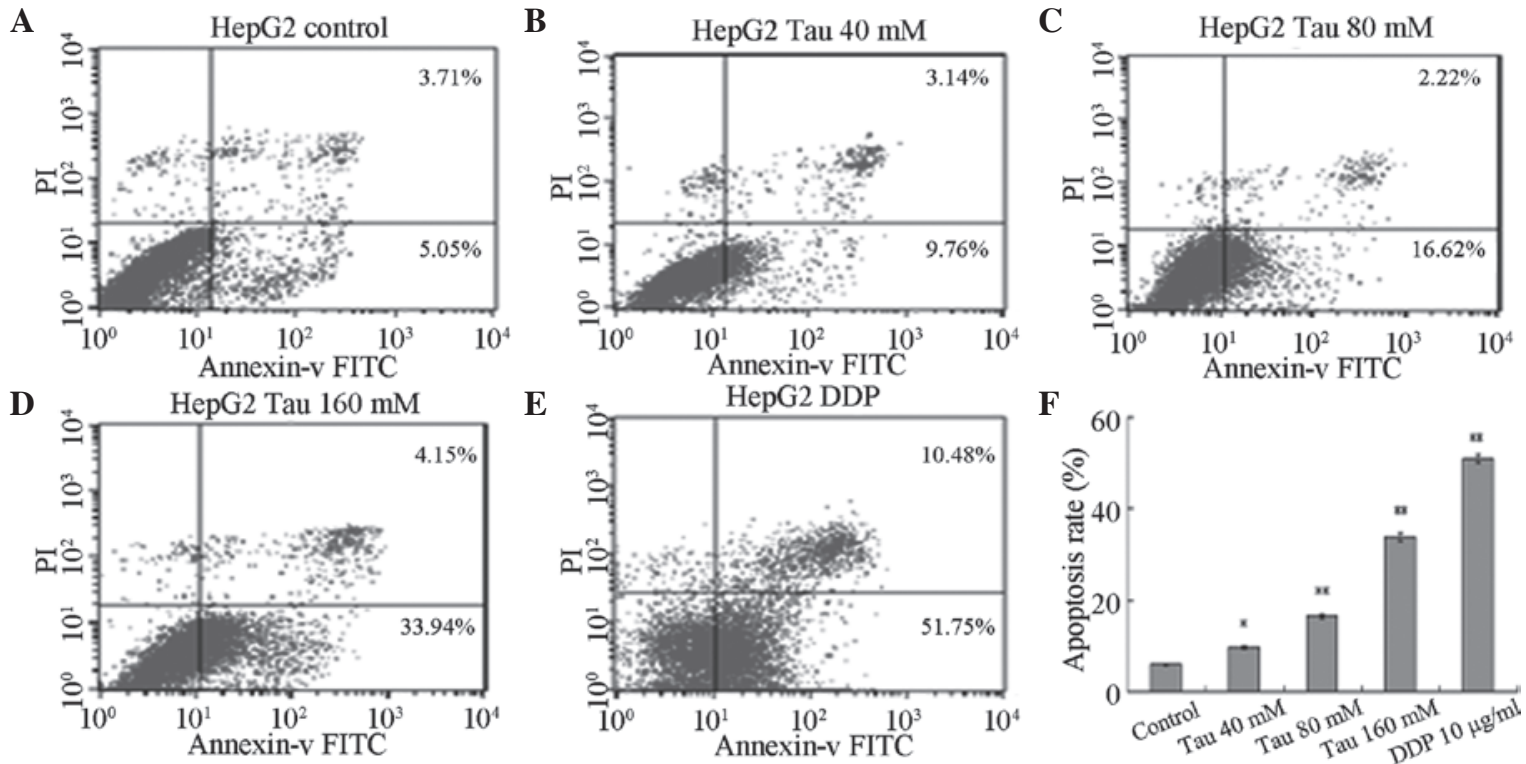

Figure 2. Effect of Tau on apoptosis induction in human hepatocellular carcinoma HepG2 cells. Effect of Tau on the apoptosis induction in human hepatocellular carcinoma HepG2 cells. HepG2 cells were treated with 40, 80, and $160 \mathrm{mM}$ Tau and $10 \mu \mathrm{g} / \mathrm{ml}$ DDP for $48 \mathrm{~h}$, and apoptotic cells were analyzed by flow cytometry. A, control group; B, $40 \mathrm{mM}$; C, $80 \mathrm{mM}$; D, $160 \mathrm{mM}$ Tau; E, 10 $\mu \mathrm{g} / \mathrm{ml}$ DDP and F, The statistical analysis of the apoptosis rate of HepG2 cells. Data from triplicate experiments were collected on a histogram $(n=4) .{ }^{*} \mathrm{P}<0.05$ and ${ }^{* *} \mathrm{P}<0.01$, vs. control group. Tau, taurine; PI, propidium iodide; FITC, fluorescein isothiocyanate; DDP, cisplatin.

and 5'-AUGAGAUUGUACAGGACCCTT-3', were designed and synthesized by Shanghai GenePharma Technology Co., Ltd. (Shanghai, China). Subsequent to the application of Lipofectamine 2000 for the transfection of the PUMA siRNA (si-PUMA) into the HHCC HepG2 cells, the effect of cell apoptosis and protein expression was observed. The following four study groups were used in this experiment. In the control group, the initial medium was discarded and replaced with a serum-free medium, which was used for cell cultivation for $6 \mathrm{~h}$. Subsequently, the cells were cultivated in complete medium for an additional $48 \mathrm{~h}$. In the negative control (NC) group, the cells were transfected with $\mathrm{NC}$ siRNA, and then cultivated in the same manner as the control group. In the si-PUMA group, the cells were transfected with the si-PUMA fragment, after which the cells underwent cultivation in the same manner as the control group. In the si-PUMA + Tau $160 \mathrm{mM}$ group, following transfection with the si-PUMA fragment for $6 \mathrm{~h}$, the cells were cultured for $48 \mathrm{~h}$ with complete medium containing $160 \mathrm{mM}$ Tau. In the Tau $160 \mathrm{mM}$ group, the cells were treated with complete medium containing $160 \mathrm{mM}$ for $48 \mathrm{~h}$.

Statistical analysis. Experimental results are presented as the mean \pm standard deviation, and statistical analysis was performed using single factor analysis of variance with SPSS statistical software (version 17.0; SPSS, Inc., Chicago, IL, USA). $\mathrm{P}<0.05$ was considered to indicate a statistically significant difference.

\section{Results}

Inhibitory effect of Tau on cell proliferation. HHCC HepG2 cells were treated with variable concentrations of Tau, and the subsequent effect of this treatment on tumor cell proliferation 
A

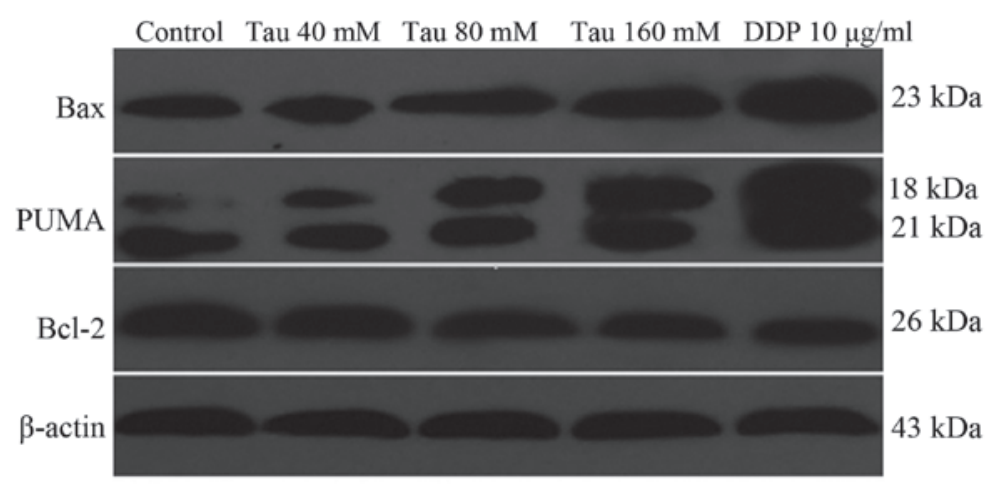

B

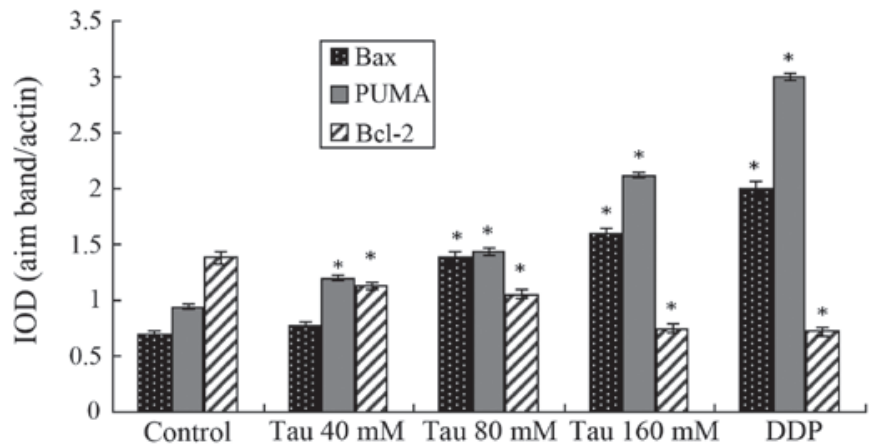

Figure 3. Protein expression levels of PUMA, Bax and Bcl-2 in human hepatocellular carcinoma HepG2 cells. (A) Representative western blot showing the protein expression of PUMA, Bax and Bcl-2. (B) Relative protein expression levels of PUMA, Bax and Bcl-2, as assessed by the gray values. The PUMA antibody also cross-reacted with an $18 \mathrm{kDa}$ band of unknown origin. Expression levels were normalized against the value obtained for $\beta$-actin protein expression. Data are expressed as the mean \pm standard deviation $(n=3)$. "P $<0.05$, vs. control group. Tau, taurine; DDP, cisplatin; PUMA, p53 upregulated modulator of apoptosis; Bcl-2, B-cell lymphoma-2; Bax, Bcl-2-associated X; IOD, optical density.

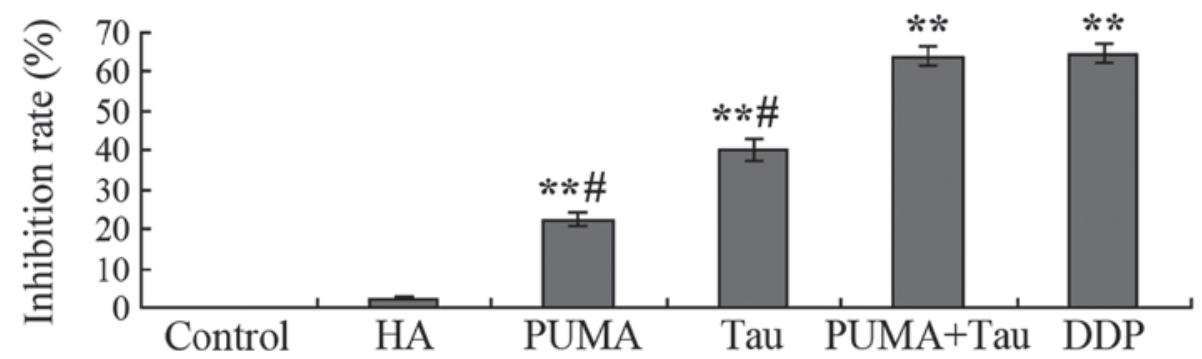

Figure 4. Inhibition rate of HepG2 cell proliferation in each group. ${ }^{* *} \mathrm{P}<0.01$, vs. control group; ${ }^{*} \mathrm{P}<0.05$, vs. DDP group. Tau, taurine; HA, pCEP4-(HA) 2 -C1; PUMA, p53 upregulated modulator of apoptosis; DDP, cisplatin.

was observed. As shown in Fig. 1, increasing the concentration and exposure time of Tau resulted in a gradual increase in the inhibition of cell proliferation in the HHCC HepG2 cells. Thus, the inhibitory effect of Tau on cell proliferation was demonstrated to be time- and dose-dependent.

Effect of Tau on apoptosis induction. HHCC HepG2 cells were cultured for $48 \mathrm{~h}$ in media containing variable concentrations of Tau $(0 \mathrm{mM}$ for the control, 40,80 and $160 \mathrm{mM}$ for the experimental groups) and DDP $(10 \mu \mathrm{g} / \mathrm{ml})$. Annexin V-FITC/PI was used to double-stain the cells. As the Tau concentration increased, the apoptosis rate of HHCC HepG2 cells (annexin $\mathrm{V}-\mathrm{FITC}^{+} / \mathrm{PI}^{-}$, right lower limit) was shown to gradually increase (Fig. 2). Statistically significant differences were observed between the apoptotic rates of the experimental groups and the control group $(\mathrm{P}<0.05$ for the $40 \mathrm{mM}$ group and $\mathrm{P}<0.01$ for the 80 and $160 \mathrm{mM}$ groups).
Effect of Tau on the protein expression levels of $P U M A, B a x$ and $B c l-2$. Western blotting results demonstrated that as the Tau concentration increased, the protein expression levels of PUMA and Bax significantly increased in the HHCC HepG2 cells, whereas the protein expression levels of Bcl-2 significantly decreased (Fig. 3). Thus, Tau appeared to have a concentration-dependent effect on the changes in PUMA, Bax and Bcl-2 protein expression levels, and these differences were statistically significant $(\mathrm{P}<0.05)$.

Effect of exogenous PUMA gene transfection on the Tau-induced inhibition of cell proliferation. Following transfection with the exogenous PUMA gene or the combined effect of the PUMA gene and Tau on HepG2 cells over 48 h cell proliferation was assessed. The results demonstrated that a significant in vitro inhibitory effect on HepG2 cell growth was observed in the PUMA gene transfection group and the PUMA + Tau group 
A

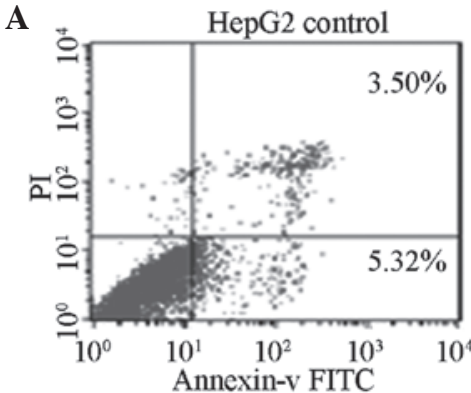

D

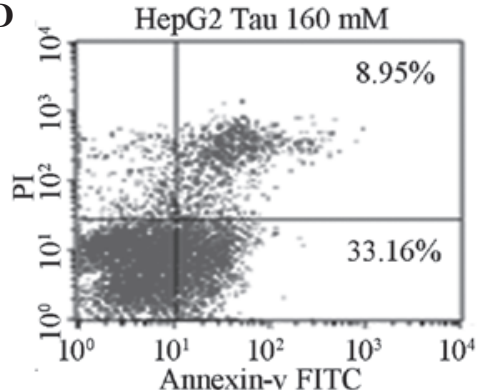

B
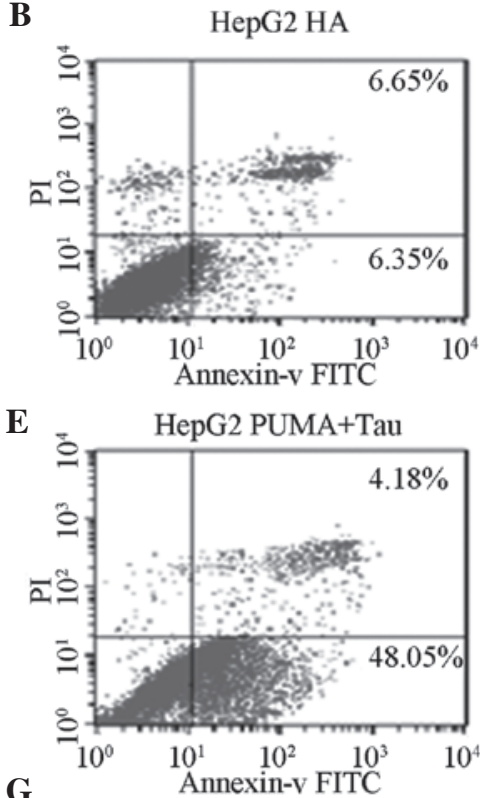

C

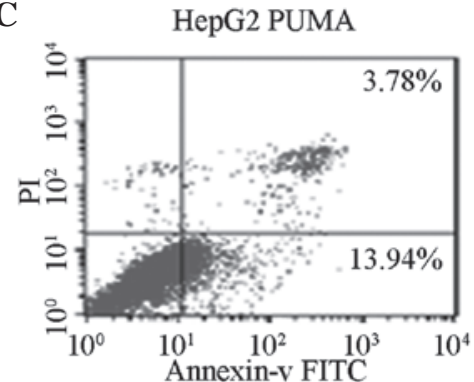

$\mathbf{F}$

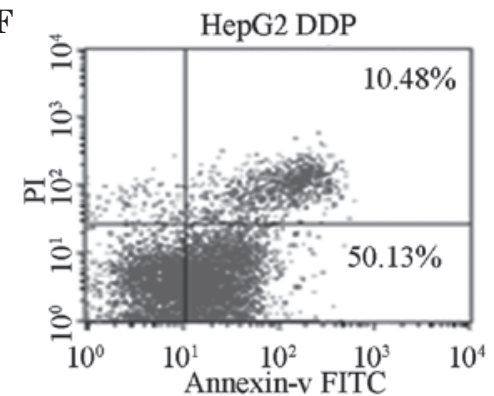

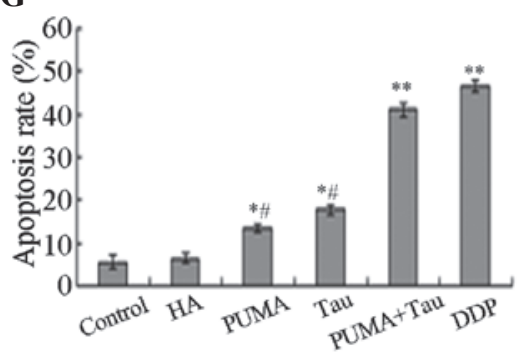

Figure 5. Effect of exogenous gene transfection with PUMA on Tau-induced apoptosis of HepG2 cells. Apoptotic cells of HepG2 were analyzed by flow cytometry. (A) control; (B) HA; (C) PUMA; (D) $160 \mathrm{mM}$ Tau; (E) PUMA + Tau group; (F) 10 $\mu \mathrm{g} / \mathrm{ml}$ DDP and (G) the statistical analysis of the apoptosis rate of HepG2 cells. Data from triplicate experiments were collected on a histogram $(n=4)$. ${ }^{*} \mathrm{P}<0.05$ and ${ }^{* *} \mathrm{P}<0.01$, vs. control or $\mathrm{HA}$ groups; ${ }^{*} \mathrm{P}<0.05$, vs. DDP group. Tau, taurine; DDP, cisplatin; PI, propidium iodide; FITC, fluorescein isothiocyanate; PUMA, p53 upregulated modulator of apoptosis; HA, pCEP4-(HA) 2 -C1.

$(\mathrm{P}<0.01)$, with the inhibition rate of cell proliferation considerably more significant in the PUMA + Tau group (Fig. 4). No statistically significant difference was observed in the growth rates between the negative control (pCEP4-(HA) $2-\mathrm{Cl})$ group and the control group $(\mathrm{P}>0.05)$. Thus, the results revealed that the inhibition rate of Tau combined with PUMA was higher, as compared with PUMA gene transfection or Tau treatment only.

Effect of exogenous PUMA gene transfection on HepG2 cell apoptosis. Following transfection with exogenous PUMA and treatment with Tau for $48 \mathrm{~h}$, annexin V-FITC/PI was used to double-stain the cells, and FCM was used to detect the cell apoptosis rate of each group. The results revealed that the apoptosis rate in each experimental group was significantly increased and statistically significant when compared with the control and HA groups ( $\mathrm{P}<0.05$; Fig. 5). The apoptosis rate of PUMA + Tau cells was significantly higher, as compared with the cells from the other groups $(\mathrm{P}<0.01)$, however there was no obvious difference compared with the DDP group. Thus, the results indicated that HepG2 cell apoptosis can be induced following transfection with the PUMA gene, and that a PUMA + Tau combination is more efficient in achieving cell apoptosis compared with the solitary use of PUMA gene transfection or treatment with Tau. The apoptosis rates of the HepG2 cells treated with Tau or PUMA gene transfection alone were significantly lower compared with that in the DDP group $(\mathrm{P}<0.05)$.

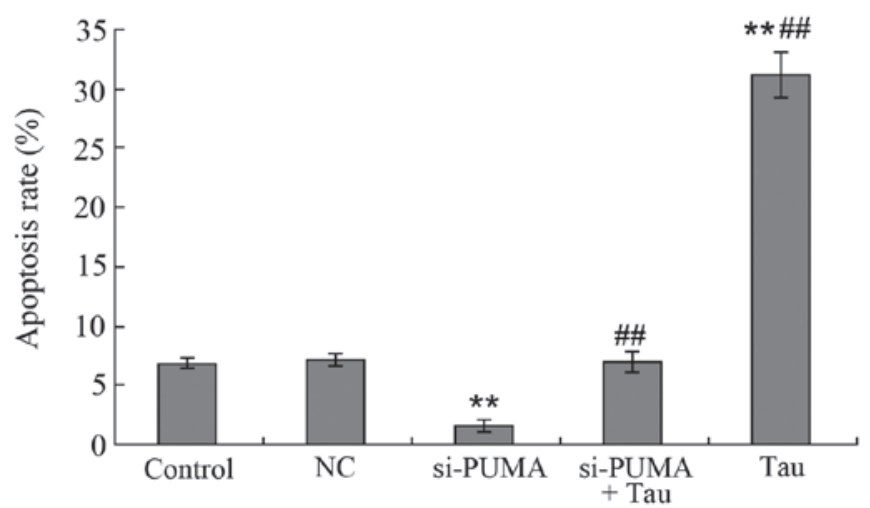

Figure 6. Effect of PUMA-specific siRNA on Tau-induced apoptosis of HepG2 cells. Apoptotic cells of HepG2 were analyzed by flow cytometry. A, control; B, NC; C, si-PUMA; D, si-PUMA + Tau and E, Tau group. Data from triplicate experiments were collected on a histogram $(n=4){ }^{* *} \mathrm{P}<0.01$, vs. control; ${ }^{\#} \mathrm{P}<0.01$, vs. si-PUMA group. FITC, fluorescein isothiocyanate; PUMA, p53 upregulated modulator of apoptosis; Tau, taurine; PI, propidium iodide; $\mathrm{NC}$, negative control; si, small interfering.

Effect of PUMA-specific siRNA on apoptosis induction. Following the specific silencing of PUMA gene expression in the HepG2 cells, the HepG2 cells were treated with $160 \mathrm{mM}$ Tau for $48 \mathrm{~h}$ and the cell apoptosis rate was determined (Fig. 6). When compared with the control group $(6.86 \pm 0.22 \%)$, the apoptosis rate of the $160 \mathrm{mM}$ Tau group $(31.16 \pm 1.86 \%)$ 
A

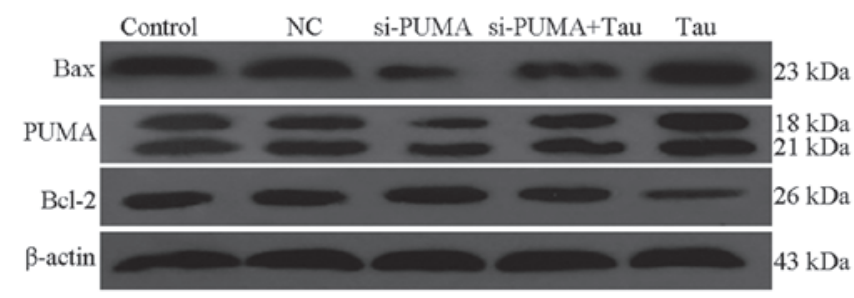

B

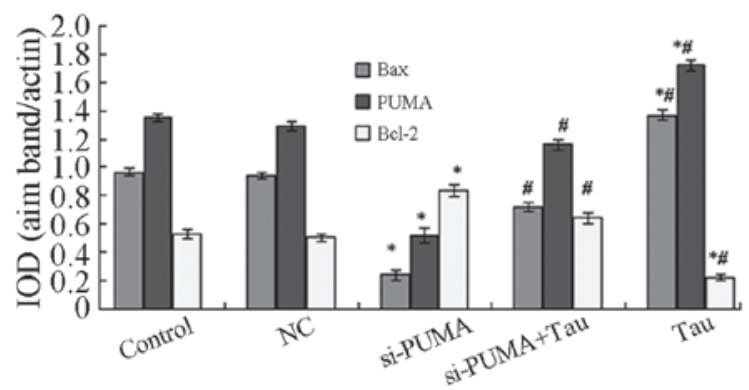

Figure 7. Effect of PUMA-specific siRNA on the protein expression levels of Bax and Bcl-2 in HepG2 cells. (A) Representative western blot showing the protein expression of PUMA, Bax and Bcl-2. (B) Relative protein expression levels of PUMA, Bax and Bcl-2, as assessed by the gray values. The PUMA antibody also cross-reacted with an $18 \mathrm{kDa}$ band of unknown origin. Expression levels were normalized against the value obtained for $\beta$-actin protein expression. Data are expressed as the mean \pm standard deviation $(n=3)$. $\mathrm{P}<0.05$, vs. control group; ${ }^{\sharp} \mathrm{P}<0.05$, vs. si-PUMA group. PUMA, p53 upregulated modulator of apoptosis; Bcl-2, B-cell lymphoma 2; Bax, Bcl-2-associated X protein; Tau, taurine; IOD, integrated optical density; NC, negative control; si, small interfering.

was significantly increased, whereas that of the si-PUMA group $(1.60 \pm 0.04 \% ; \mathrm{P}<0.01)$ was significantly decreased. No statistically significant difference was observed in the apoptosis rate between the NC group $(7.15 \pm 0.27 \%)$ and the si-PUMA + Tau group $(7.02 \pm 0.25 \% ; \mathrm{P}>0.05)$. However, when compared with the si-PUMA group $(1.60 \pm 0.04 \%)$, the apoptosis rate of the si-PUMA + Tau group $(1.60 \pm 0.04 \%)$ was significantly increased $(\mathrm{P}<0.01)$.

Effect of PUMA-specific siRNA on the protein expression levels of Bax and Bcl-2. Following the specific silencing of PUMA expression in the HHCC HepG2 cells, Bax and Bcl-2 protein expression levels in the cells were shown to significantly change (Fig. 7). The results revealed that the protein expression levels of PUMA and Bax in the si-PUMA group were significantly downregulated, whereas Bcl-2 protein expression was significantly upregulated. These changes were statistically significant $(\mathrm{P}<0.05)$. When compared with the si-PUMA group, PUMA and Bax protein expression levels in the si-PUMA + Tau group were found to be significantly upregulated, whereas the expression of $\mathrm{Bcl}-2$ protein was significantly downregulated $(\mathrm{P}<0.05)$. The protein expression levels of PUMA, Bax and Bcl-2 in the NC group did not show a statistically significant difference from those in the control group $(\mathrm{P}>0.05)$. These results indicated that Tau was able to increase the protein expression levels of PUMA, which may be a possible mechanism through which Tau is able to induce HHCC HepG2 cell apoptosis.

\section{Discussion}

Liver cancer is a digestive system neoplasm with high mortality and recurrence rates. This cancer type is the fifth most common malignancy and the third most common cause of cancer mortality worldwide (16). Clinical studies have shown that numerous chemotherapeutic drugs used to treat liver cancer have strong toxic adverse effects. The insensitivity of tumor cells to chemotherapeutic drugs and the toxic adverse effects of these drugs have detrimental impacts on clinical efficacy. Previous studies have found that Tau possesses broad physiological and pharmacological functions, and previous research has shown that changes in the in vivo concentration of Tau may be a predictor of cancer $(11,12)$. A high concentration of Tau may appear in the urine of patients with non-muscular invasive bladder cancer, suggesting that Tau may become a new indicator for the diagnosis of bladder cancer. Research by Nagy et al (17) has shown that the Tau derivative, glutaurine, significantly decreases the occurrence of MC29/1 virus-induced HHCC and leukemia in turkey chicks. In addition, McCourt et al (18) demonstrated that the Tau derivative, taurolidine, can inhibit carcinoma growth in vitro and in vivo. Research by Reddy et al (19) has shown that Tau can inhibit colorectal cancer by increasing the activity of glutathione transferase, NAD $(\mathrm{P}) \mathrm{H}$ reductase and glucuronyl transferase.

The present study found that Tau, at a concentration between 20 and $160 \mathrm{mM}$, exerts an inhibitory effect on HHCC HepG2 cell proliferation and apoptosis induction, with the effect time- and dose-dependent (Figs. 1 and 2). With increasing Tau concentrations, the protein expression levels of PUMA and Bax in the HHCC HepG2 cells were found to be significantly upregulated, whereas the protein expression of Bcl-2 was significantly downregulated (Fig. 3). In addition, the effect of Tau on the protein expression levels of PUMA, Bax and Bcl-2 was found to be dose-dependent. Exogenous PUMA gene transfection significantly increased the effect of Tau on HepG2 cell apoptosis and proliferation inhibition. Application of PUMA gene transfection and Tau treatment was shown to be as effective as treatment with DDP (with a final concentration of $10 \mathrm{mg} / \mathrm{ml}$; Figs. 4 and 5). However, following the targeted silencing of PUMA expression in HepG2 cells and Tau treatment for $48 \mathrm{~h}$, the apoptosis rate of the si-PUMA + Tau group was shown to be significantly lower compared with that of the Tau group (Fig. 6). Furthermore, when compared with the si-PUMA group, the protein expression levels of PUMA and Bax in the si-PUMA + Tau group were significantly upregulated, while $\mathrm{Bcl}-2$ protein expression was significantly downregulated $(\mathrm{P}<0.05)$. These results demonstrated that Tau has the combined effect of upregulating PUMA and Bax protein expression, while downregulating $\mathrm{Bcl}-2$ protein expression (Fig. 7). Through this important mechanism, Tau can induce apoptosis and inhibit the proliferation of HHCC HepG2 cells. Therefore, PUMA plays an important role in the anti-HHCC 
effect of Tau, and may become a novel target for use in gene therapy against liver cancer.

Tau can inhibit tumor proliferation through several mechanisms. Firstly, Tau has been hypothesized to enhance antioxidation in the body through the removal of strong oxidizing agents, which subsequently protects normal cells from oxidative damage and induces tumor cell apoptosis $(9,20,21)$. For example, Tau can reverse the morphine-induced downregulation of Bcl-2 and effectively control morphine-induced oxidative damage, thus preventing nerve cell toxicity (22). In B16F10 mouse melanoma cells, Tau functions by upregulating superoxide dismutase genes, glutathione peroxidase and catalase, while inhibiting cell growth by decreasing the concentration of reactive oxygen species in a dose-dependent manner (23). In addition, following treatment with Tau for liver fibrosis, the levels of hydrogen peroxide lipids, transforming growth factor- $\beta$ and hydroxyproline in the blood and liver are significantly reduced, and liver damage and fibrosis are decreased (24).

Secondly, Tau may inhibit tumor proliferation by enhancing immunity. Abd-Rabou et al (25) found that application of Tau with curcumin promotes immunity in an organism, which subsequently resulted in the dissolution of Huh-7 carcinoma cells.

Thirdly, Tau may exert an antitumor function by increasing the efficacy and decreasing the toxicity of chemotherapeutic drugs. Following intravenous injection or gastric perfusion of Tau and/or cyclophosphamide (CTX) in tumor-bearing mice, the inhibition rate of the combination drug group was found to be much higher compared with that of the CTX-only group, indicating that Tau enhances the efficacy of CTX chemotherapeutic drugs (15). The same method was used to treat Lewis liver tumor-bearing mice, where Tau was demonstrated to increase the white blood cell count, leading to the alleviation of CTX toxicity (26). Furthermore, the effect of Tau on the apoptosis of human cervical carcinoma cells has been demonstrated to be time- and dose-dependent, and the apoptosis rate has been found to be more significant when combined with DDP.

In addition to modulating the expression of $\mathrm{p} 53$, Tau can activate caspases $3,6,7$ and 9 to activate the mitochondrial pathway (27). Furthermore, Tau has been shown to mitigate the oxidative stress and cell damage produced by arsenic through mitochondrial-dependent and independent pathways (28). Zhang et al (29) found that downregulation of nicotinamide $\mathrm{N}$-methyltransferase leads to the upregulation of PUMA gene expression and the induction of apoptosis in breast carcinoma cells via the induction of mitochondrial apoptotic pathways. In mouse carcinoma cell models, PUMA expression has been shown to increase significantly following inactivation of human epidermal growth factor receptor 2 (HER2). However, reduced PUMA expression may result in a decrease in caspase activation, which leads to a reduction in the apoptosis rates of mouse breast carcinoma cells caused by HER 2 inactivation, and lung carcinoma cells induced by a mutation in the epidermal growth factor receptor (30). The results of the present study demonstrated that Tau was able to downregulate the expression of the apoptosis-inhibiting protein, Bcl-2, while upregulating the expression of the apoptosis-inducing proteins, PUMA and Bax, to subsequently alter the $\mathrm{Bax} / \mathrm{Bcl}-2$ ratio and induce apoptosis of carcinoma cells through a mitochondrial-dependent pathway. PUMA was also demonstrated to play a key role in this process.

In conclusion, the PUMA gene plays an important role in mechanism underlying the effect of Tau on cell proliferation inhibition and the induction of apoptosis in HHCC HepG2 cells. Therefore, the PUMA gene may become a novel target for use in gene therapy against liver cancer. However, whether PUMA is the only Tau-induced apoptosis-associated gene requires further study.

\section{References}

1. Shen YM, Yu SF and Cui MY: The impact and learning on the farmers' health when conducting health education in rural communities. Zhongguo NongCun Wei Sheng Shi Ye Guan Li 27: 603-605, 2007.

2. Subramaniam A, Shanmugam MK, Perumal E, et al: Potential role of signal transducer and activator of transcription (STAT)3 signaling pathway in inflammation, survival, proliferation and invasion of hepatocellular carcinoma. Biochim Biophys Acta 1835: 46-60, 2013.

3. Sia D and Villanueva A: Signaling pathways in hepatocellular carcinoma. Oncology 81: 18-23, 2011.

4. Parkin DM, Bray F, Ferlay J and Pisani P: Global cancer statistics, 2002. CA Cancer J Clin 55: 74-108, 2005.

5. Ho M: Advances in liver cancer antibody therapies: a focus on glypican-3 and mesothelin. BioDrugs 25: 275-284, 2011.

6. Lin J, Huang S, Wu S, et al: MicroRNA-423 promotes cell growth and regulates $\mathrm{G}(1) / \mathrm{S}$ transition by targeting $\mathrm{p} 21 \mathrm{Cip} 1 / \mathrm{Waf} 1$ in hepatocellular carcinoma. Carcinogenesis 32: 1641-1647, 2011.

7. Huxtable RJ: Physiological actions of taurine. Physiol Rev 72: 101-163, 1992.

8. Wójcik OP, Koenig KL, Zeleniuch-Jacquotte A, Costa M and Chen Y: The potential protective effects of taurine on coronary heart disease. Atherosclerosis 208: 19-25, 2010.

9. Wang L, Zhao N, Zhang F, Yue W and Liang M: Effect of taurine on leucocyte function. Eur J Pharmacol 616: 275-280, 2009.

10. Yu JS and Kim AK: Effect of combination of taurine and azelaic acid on antimelanogenesis in murine melanoma cells. J Biomed Sci 17 (Suppl 1): S45, 2010.

11. El Agouza IM, Eissa SS, El Houseini MM, El-Nashar DE and Abd El Hameed OM: Taurine: a novel tumor marker for enhanced detection of breast cancer among female patients. Angiogenesis 14: 321-330, 2011.

12. Srivastava S, Roy R, Singh S, et al: Taurine - a possible fingerprint biomarker in non-muscle invasive bladder cancer: A pilot study by 1H NMR spectroscopy. Cancer Biomark 6: 11-20, 2010.

13. Neary PM,Hallihan P, Wang JH,PfirrmannRW,Bouchier-Hayes DJ and Redmond HP: The evolving role of taurolidine in cancer therapy. Ann Surg Oncol 17: 1135-1143, 2010.

14. Zhang X, Sheng J, Zhang C, et al: Taurine induces apoptosis in pulmonary artery smooth muscle cells. Zhongguo Zhong Yao Za Zhi 37, 654-657, 2012.

15. Zhang X, Tu S, Wang Y, Xu B and Wan F: Mechanism of taurine-induced apoptosis in human colon cancer cells. Acta Biochim Biophys Sin (Shanghai) 46: 261-272, 2014.

16. Giacomin A, Sergio A, Vanin V, Gazzola A, Cazzagon N and Farinati F: Molecular targeted therapy in hepatocellular carcinoma: present achievements and future challenges. Dig Dis 30: 284-288, 2012.

17. Nagy K, Bátkai L, Tálas M and Feuer L: Effect of glutaurine on liver tumour development and acute leukaemia induced by MC29 virus in turkey poults. Acta Microbiol Hung 30: 37-42, 1983.

18. McCourt M, Wang JH, Sookhai S and Redmond HP: Taurolidine inhibits tumor cell growth in vitro and in vivo. Ann Surg Oncol 7: 685-691, 2000.

19. Reddy BS, Rao CV, Rivenson A and Kelloff G: Chemoprevention of colon carcinogenesis by organosulfur compounds. Cancer Res 53: 3493-3498, 1993.

20. Das J, Ghosh J, Manna P and Sil PC: Taurine suppresses doxorubicin-triggered oxidative stress and cardiac apoptosis in rat via up-regulation of PI3-K/Akt and inhibition of p53, p38-JNK. Biochem Pharmacol 81: 891-909, 2011. 
21. Mates JM, Segura JA, Alonso FJ and Marquez J: Sulphur-containing non enzymatic antioxidants: therapeutic tools against cancer. Front Biosci (Schol Ed) 4: 722-748, 2012.

22. Zhou J, Li Y, Yan G, et al: Protective role of taurine against morphine-induced neurotoxicity in C6 cells via inhibition of oxidative stress. Neurotox Res 20: 334-342, 2011.

23. Yu J and Kim AK: Effect of taurine on antioxidant enzyme system in B16F10 melanoma cells. Adv Exp Med Biol 643: 491-499, 2009.

24. Miyazaki T, Karube M, Matsuzaki Y, Ikegami T, Doy M, Tanaka $\mathrm{N}$ and Bouscarel $\mathrm{B}$ : Taurine inhibits oxidative damage and prevents fibrosis in carbon tetrachloride-induced hepatic fibrosis. J Hepatol 43: 117-125, 2005.

25. Abd-Rabou AA, Zoheir KM and Ahmed HH: Potential impact of curcumin and taurine on human hepatoma cells using Huh-7 cell line. Clin Biochem 45: 1519-1521, 2012.

26. Wang L, Zhao N, Zhang F, Yue W and Liang M: Effect of taurine on leucocyte function. Eur J Pharmacol 616: 275-280, 2009.
27. Kim T and Kim AK: Taurine enhances anticancer activity of cisplatin in human cervical cancer cells. Adv Exp Med Biol 776: 189-198, 2013.

28. Das J, Ghosh J, Manna P, Sinha M and Sil PC: Taurine protects rat testes against $\mathrm{NaAsO}(2)$-induced oxidative stress and apoptosis via mitochondrial dependent and independent pathways. Toxicol Lett 187: 201-210, 2009.

29. Zhang J, Wang Y, Li G, Yu H and Xie X: Down-regulation of nicotinamide $\mathrm{N}$-methyltransferase induces apoptosis in human breast cancer cells via the mitochondria-mediated pathway. PLoS One 9: e89202, 2014.

30. Bean GR, Ganesan YT, Dong Y, et al: PUMA and BIM are required for oncogene inactivation-induced apoptosis. Sci Signal 6: ra20, 2013. 